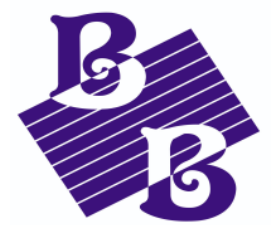

BioBacta

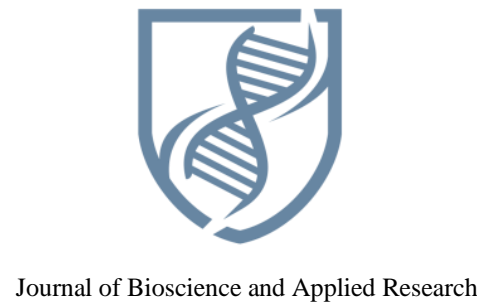

www.jbaar.org

\title{
Standpoints on immune responses against COVID-19
}

\author{
Mabrouk A. Abo-Zaid ${ }^{1}$, Ahmed H. Ismail ${ }^{1}$, Ali H Amin ${ }^{2,3}$ \\ 1: Biology department, faculty of science, Jazan University, Jazan, Saudi Arabia \\ 2: Deanship of Scientific Research, Umm Al-Qura University, Makkah, Saudi Arabia; \\ 3: Zoology Department, faculty of science, Mansoura University, Mansoura, Egypt; \\ mabrouk_ss@yahoo.com; 00966560963725 \\ ahanafy12@gmail.com; 00966582911612 \\ alihamin@gmail.com; 00966595545502
}

Running Title: The immune response against COVID-19

\begin{abstract}
COVID-19 pandemic disease has firstly occurred in Wuhan, Hubei, China. Then the infection spread globally. Many researchers now suggest the strong similarities of COVID-19 with the previous pathogenic human beta coronaviruses SARS-CoV and MERS-CoV. But there is limited knowledge about the immune response to COVID-19. So on the basis of the immune response of these viruses, we will try to predict how the immune system can deal with this infection. In this review, we focus on human coronavirus types and discuss current knowledge of immune response against SARS-CoV and MERS$\mathrm{CoV}$ immune response, which may be helpful in offering the perspectives on immune responses against COVID-19.
\end{abstract}

Key words: Coronavirus, COVID-19, SARS-CoV-2, immune response, cytokine storm.

Received: August 18, 2020. Accepted: September 5, 2020. Published: September 17, 2020

DOI: $10.21608 /$ JBAAR.2020.113160 


\section{Introduction}

Coronaviruses are classified as a genus of the family coronaviridae, which contains variable pathogens of mammals and birds causing different types of diseases like pneumonia, enteritis, polyserositis, hepatitis, nephritis, and many other disorders. Coronavirus and coronavirus-like infections have been investigated in swine, cattle, horses, cats, dogs, rats, birds, bats, rabbits, ferrets, mink, and various wildlife species, although many coronavirus infections are subclinical or asymptomatic (Maclachlan and Dubovi, 2010).

\section{Human Coronavirus Types}

Coronaviruses have their name from the crown-like proteins on their outer surface (spike proteins). They are classified into four main subgroups (alpha, beta, gamma, and delta). Human coronaviruses were firstly detected in the 1960s from which seven types were identified for their ability to infect people (229E, NL63, OC43, HKU1, MERS-CoV, SARS-CoV and SARS-CoV2 and the newly detected one in 2019 called COVID-19). From these viruses 229E, NL63, OC43, and HKU1 were registered as human coronaviruses, however recently some animal coronaviruses proved its ability to infect people and make them sick these are SARS-CoV, MERS-CoV, and COVID-19 (Killerby et al., 2018; Xiao et al., 2020).

\section{Immune response against SARS:}

In SARS infection, the laboratory manifestations include lymphopenia, neutrophilia, thrombocytopenia, and high levels in serum lactate dehydrogenase(LDH), glutamic pyruvic transaminase (GPT), and creatine kinase(CK) Cheng et al. (2007). Lymphopenia is observed in most SARS patients (>98\%) in different studies, and most of them have reduced T-helper
(CD4+) and T-cytotoxic/suppressor (CD8+) cell counts during the $1^{\text {st }}$ period of infection ; these values fall down to their lowest point from day 5 to 7 from disease onset (fever) and restore gradually during the recovery phase (He et al., 2005; Lam et al., 2004) figure (1) shows Innate immune response regulation is accompanying with the improvement of adaptive immunity and disease severity in SARS-CoV infection. Particularly, SARS has showing indirect strategies to overwhelm antiviral interferon type I (INF) responses in infected cells $\mathbf{L i}$ and $\mathbf{X u}$ (2010). Infected tissues showed an upregulation of pro-inflammatory cytokines/chemokines (IL-6, IP-10, and MCP-1) as an inflammatory response, also heavy infiltration of macrophages occurred in these infected cells (Astuti et al., 2020; Huang et al., 2020b). SARS-CoV infection is characterized by a significant increase in IFN- $\gamma$ levels which is a Th1 cytokine responsible for cell mediated-immunity and fighting intracellular pathogens while reduction in IL-4 is well observed which stimulates the humoral immunity that suppresses extracellular microbial infections. Schulze et al. (2008). There is also an up-regulation of proinflammatory cytokines, such as TNF alpha, IL-1ß, and IL-6, which might enhance disease outcome Xie et al. (2003). SARS-CoV infection could induce monocytes/macrophages, dendritic cells, and lung epithelial cells to produce IL-1b, IL-6, IL8, IP-10, and MCP-1 Li and Xu (2010). Based on patient's case reports during the first two weeks of illness onset, levels of IL1b, IL-6, IL-12, IFN-g, IL-8, IP-10, and MCP-1 are markedly upregulated; a subsequent decrease in the concentration of these cytokines is accompanying with recovery from SARS pneumonia. IL-6 has been powerfully related to radiographic score, patients with a lethal outcome have been increased $\mathrm{Th} 2$ cytokines Li et al. (2008). 


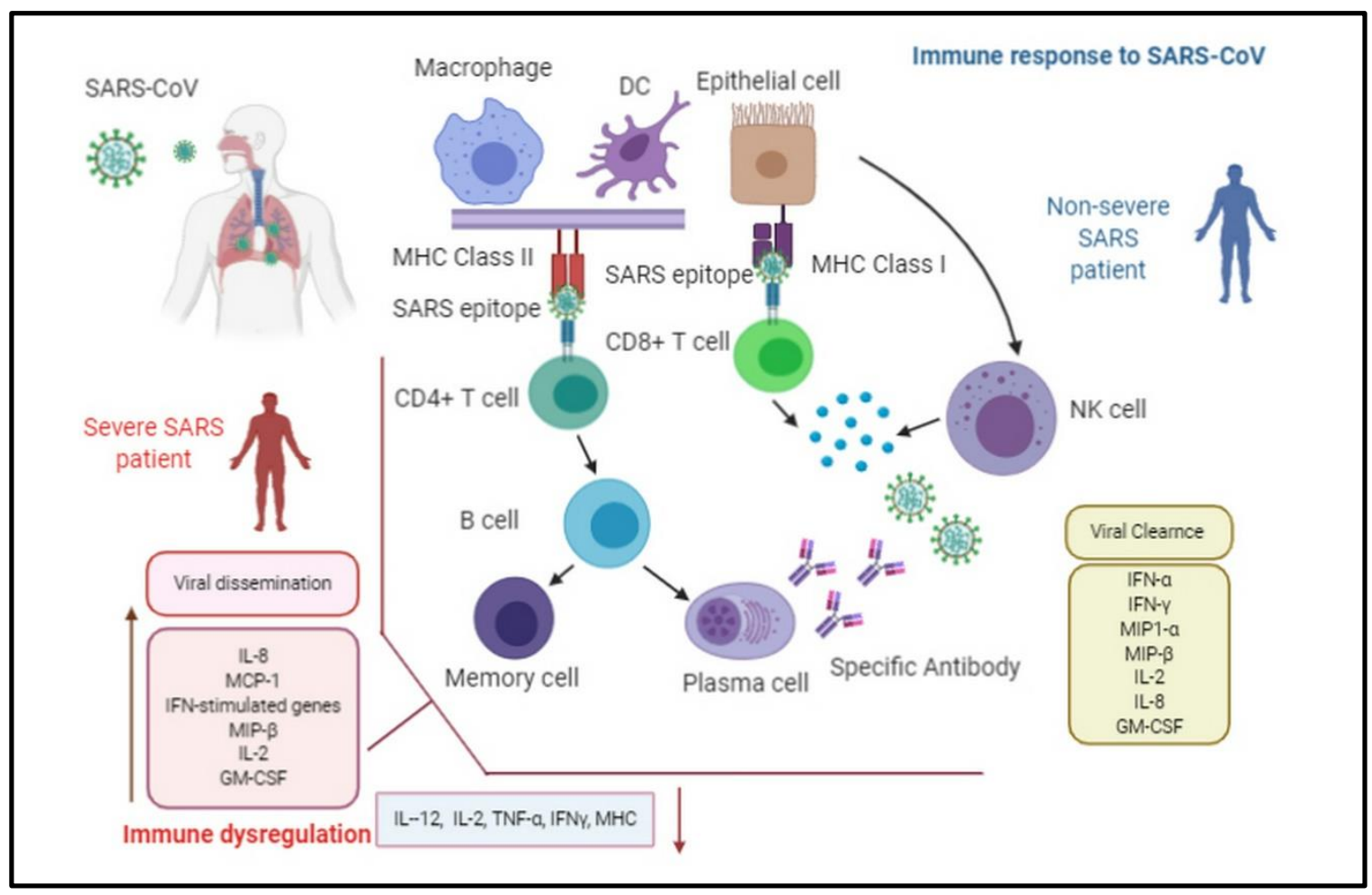

Fig. 1: SARS-CoV infected epithelial cells represents the SARS epitope by MHC I to recruit T cytotoxic (CD8+T cell). Macrophage and dendritic cell (DC) are infected by SARS-CoV and represent SARS epitope by MHC II to recruit T helper cell (CD4+T cell) and further cytokine storm starts in severe cases. Humoral immune response enhances the production of $\operatorname{IgG}, \operatorname{IgM}$, and $\operatorname{IgA}$, and viral entrance to other cells is prevented. CD8+T cells secrete perforin and granzyme to kill virally infected cells (Viral clearance). Immune dysregulation in severe SARS patients is marked by continued expression of inflammatory chemokines and IFN-stimulated genes (ISGs) and deficiencies in MHC and immunoglobulin gene expression, IL12, IL-2. Immune-mediated pathology worsens in the minority of susceptible SARS patients and unsettled interferon $\gamma$ induced protein 10 (IP-10) expression and the viral burden is associated with the deprived result.

\section{Immune response against MERS CoV.:}

Dendritic (DCs) and macrophages (M $\Phi)$ are significant to support innate immunity and can elicit release enormous amounts of cytokines and chemokines. These cells can relocate from peripheral tissues to the lymphoid tissue to actuate the $\mathrm{T}$ cell population Crespo et al. (2013). Both $T$ \& B lymphocytes are stimulated for a specific immune response against MERS-CoV Mubarak et al. (2019).
MERS-CoV has advanced strategies to impact innate immunity and inhibit IFN releasing pathways (Balachandran et al., 2000; Mubarak et al., 2019) As showing in figure (2): Pattern recognition receptors (PRRs), for examples, Toll-like receptors (TLRs) and Retinoic Acid-Inducible gene-I-like receptors (RIG-I), are vital mediators of the innate immune response Kato et al. (2011). After TLRs recognized to the viral epitope, one of the two, unlike adaptor molecules, is 
adverse regulators of the TLR signaling pathways. This stimulation consequently results in the expression of both peroxisome proliferator-activated receptor- $\gamma$ (PPAR) and IL-1R-associated kinase (IRAK-M), which are adverse regulators of IRF7, the transcription factor that induces the gene expression of IFN- $\alpha / \beta$ (AlQahtani et al., 2017). Natural killer (NK) cells, T cytotoxic $(\mathrm{CD} 8+)$ cells, and macrophages $(\mathrm{M} \Phi)$, are stimulated, and their antiviral toxic cytokines are produced Welsh and Waggoner (2013). Antiviral cytokines called the immune response proinflammatory cytokine (for example IL-6 and TNF- $\alpha$ production) against any infection has several problems in the host, such as tissue injuries Zhou et al. (2014). The pro-inflammatory cytokine response can control viral spreading. Normally, Interferons (IFNs) play vital roles during some viral infections and can be sensitized by double-stranded RNAs (dsRNAs) Balachandran et al. (2000). The immunological response may be at first associated with interfering with the NF- $\mathrm{BB}$ signaling pathway, which is regularly responsible for the excitation of the immune response pro-inflammatory cytokine Canton et al. (2018). In a previous study presented that IFN- $\beta$ has a strong inhibitory impact on MERS-CoV in comparison to that of other tested IFNs Hart et al. (2014). Al-Qahtani et al. (2017) indicated the vital effect of the MERS spike glycoprotein (MERS-CoV-S) on the responsiveness of macrophages and monocytes through Toll-like receptor-4 (TLR-4) signaling pathways. MERS-CoV infection enhances the production of IFN- $\alpha / \beta$ by virally infected cells, which leads to the release of some chemokines, such as monocyte chemoattractant Protein-1(MCP-1), IL10, and Interferon gamma-induced protein-10 (IP-10), which are responsible for $\mathrm{T}$ cell recruitment. CD4+ helper T cells (Th1) and NK cells need signaling by IL12 and IFN- $\gamma$ for their stimulation (Zhou et al., 2015). IFN- $\gamma$ contributes to the activation of NK cells and CD8+ T cells that help clear viruses Welsh and Waggoner (2013). $\mathrm{T}$ lymphocytes are significant agents essential for immunity against viral infections; $\mathrm{T}$ helper (CD4+) cells assist virus-specific antibody production via sensitized B cells. While, T cytotoxic (CD8+) cells are cytotoxic and kill virally infected cells (Xu and Gao, 2004; Zhao et al., 2014). Furthermore, throughout the highly of MERS-CoV infection, the virus attacks the immune system and inhibits the response of $\mathrm{T}$ cells. These actions may further damage the functions of B- lymphocytes Ying et al. (2016). A new study revealed that T-helper (CD4+) cells were more affected by MERS-CoV infection. Additionally, MERS-CoV can instigate $\mathrm{T}$ cell the apoptosis by activating apoptosis process via intrinsic and extrinsic pathways Ying et al. (2016). Remarkably, there was a significant upregulation in the level of IL-17 in patients infected by MERS-CoV Mahallawi et al. (2018). T helper subset cells, especially Th17 cells, produce the pro-inflammatory cytokine IL-17 via the STAT3 and NF- $\mathrm{B}$ signaling pathways Manni et al. (2014). These results advises that MERS-CoV infection stimulates the production of Th17 cytokines. These Th17 cytokines can attract neutrophils and monocytes to the site of infection and lead to the activation of other succeeding cytokine and chemokine cascades, including IL-1, IL-6, IL-8, TNF- $\alpha / \beta$, and MCP-1. Neutralizing antibodies are very potent in neutralizing viral infectivity through blocking their entry into host cells. The detection of MERS-CoV- specific antibodies in human serum is one of the confirmatory diagnostic tests for MERS-CoV infection Mubarak et al. (2019). It is essential to determine even if these antibodies are a strong weapons of the adaptive immune response to MERS-CoV infection. However, the observation of the anti-MERS-CoV antibody response arises from 14 to 21 after infection Corman et al. (2016). 


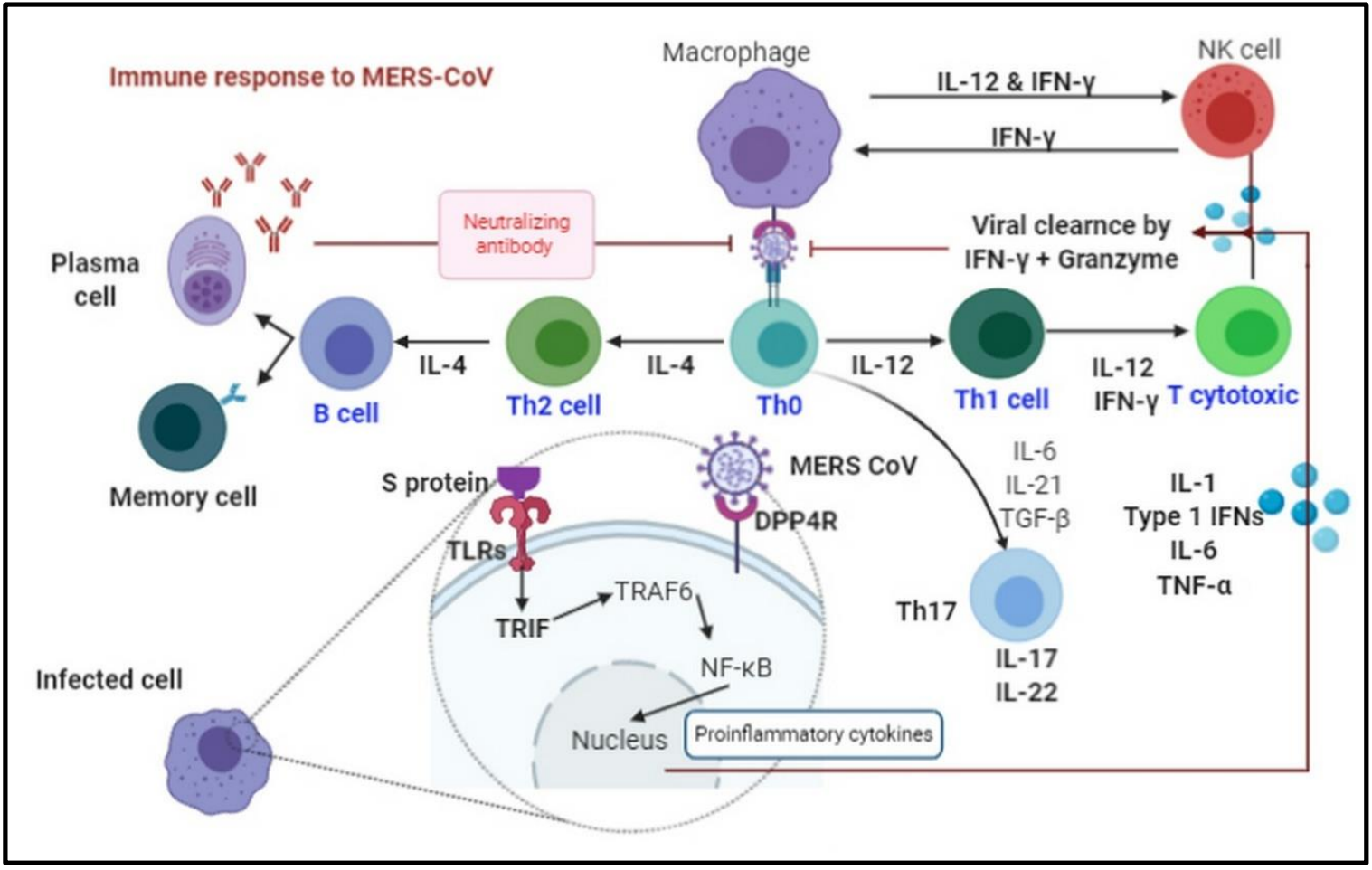

Fig. 2 describes the immune response to MERS-CoV. MERS-CoV phagocytosed by macrophages and then macrophages present MERS-CoV peptide epitopes to Th0 cells. This process leads to $\mathrm{T}$ cell activation and production of cytokines related to the different $\mathrm{T}$ cell subsets including Th1, Th2, and Th17; then cytokines released in abundant quantity to enhancing the immune response and amplification. The release of these cytokines more and more abundant form due to viral persistence has the opposite effect on CD+4 T cells, CD8+ T cells, and Natural Killer (NK) cell activation by preventing IL12 and IFN$\gamma$ secretion. Nevertheless, CD8+ T cells produce cytotoxic substances include IFN- $\gamma$ and granzyme, to kill MERS-CoV.

\section{Immune response and cytokine storm in COVID-19:}

In view of that, a full study has not been achieved on immune response against SARS-CoV 2 (COVID-19), the only method to debate this objective is mention to previous data about SARS-CoV and MERS-CoV (Fig.3). In the first after a virus attacks the host, the first response is recognized via pattern recognition receptors (PRRs) including C-type lectin-like receptors, Toll-like receptors (TLR), NOD-like receptors (NLR), and Retinoic Acid-Inducible gene-I-like receptors (RIG-I) (Addi et al., 2008; Yi et al., 2020). By different pathways, the antigen (virus) stimulate the production of inflammatory factors, maturation of dendritic cells, and synthesis of type I interferons (IFNs) which restrict the spreading of the virus and enhance macrophage phagocytosis of viral peptide epitopes (Li et al., 2020; Tufan et al., 2020). Shortly, the adaptive immunological response joins the fight against the virus. The abundant pro-inflammatory cytokines produced by an innate and adaptive immune response, play a vital role in the defense by activation of CD4+ T cells and CD8 $+\mathrm{T}$ cells, are controlling the virally replication and reducing the viral infected cells (Mathern and Heeger, 2015; Sevajol et al., 2014). The $\mathrm{T}$ helper cells (CD4+T cells) sensitize $\mathrm{B}$ lymphocytes to proliferate to plasma cells and produce viral peptide epitopes-specific antibodies, and $\mathrm{T}$ 
cytotoxic cells (CD8+Tcells) release cytotoxic factors to clearance viral infected cells immediately. Natural killer (NK) cells releases toxic substances, in a similar way to cytotoxic $T$ cells, which clearance the virallyinfected cells instantly Yi et al. (2020). CD8+ T cytotoxic cells are equipped with preformed mediators. Cytotoxic agents are deposited inside granules, in both $\mathrm{CD} 8+\mathrm{T}$ cells and NK cells, until interaction with a viral-infected cell triggers their release. One of these substances called perforin that can make pores in cell membranes; these holes let entry of other factors into a target cell to facilitate the destruction of the cell. Enzymes called granzymes are also stored in, and released from, the granules. Granzymes enter infected cells via the pores made by perforin Trapani (2001). CD4+ T cells produce pro-inflammatory to activate the defending cells. The humoral immunity including complements such as c3a and c5a and antibodies is also essential in combating the viral infection ( $\mathbf{L i}$ et al., 2020; Risitano et al., 2020). However, the complement stimulation can contribute to the maladaptive inflammatory response seen in some patients with severe COVID-19 Risitano et al. (2020). Nevertheless, COVID-19 can inhibit T-lymphocytes' functions by inducing apoptosis of T cells Tufan et al. (2020). Inflammatory cytokines produced from Th17 contributing to severe lung diseases and death rates of lower respiratory tract infections caused by coronaviruses. This comprises host inflammatory cytokine responses against COVID-19 Yang et al. (2020). The severity of COVID-19 is related to the high level of the pro-inflammatory cytokines and subsets of immune cells (Huang et al., 2020b; Wang et al., 2020).

Immunological response to SARS-CoV2 (COVID-19) like SARS-CoV is being mediated by cytokines. According to some case reports in Wuhan from SRASCoV2 patients showed an increase in Interleukin-6; neutrophil count and C-reactive protein (CRP) and total lymphocytes are decreases Astuti et al. (2020).
The finding lymphopenia in COVID-19 patients is in line with SARS-CoV and MERS-CoV patients; cytokine storm and lymphopenia may have a main role in the severity of SARS-CoV2 (Nicholls et al., 2003; Wong et al., 2004). Based on hospitalized patient reports most patients had lymphopenia and pneumonia with characteristic pulmonary ground-glass opacity changes on chest CT. (Diao et al., 2020; Prompetchara et al., 2020) reported that reduced in $\mathrm{T}$ cell counts and the surviving $\mathrm{T}$ cells appear dysfunctional in COVID-19 patients. Furthermore, from COVID-19 patients report in severe cases recorded that pro-inflammatory cytokines are increased including IL-2, IL-7, IL-10, G-CSF, IP-10, MCP-1, MIP-1A, and TNF- $\alpha$ Huang et al. (2020b) found that increased cytokines levels like IL-6, IL-10, and TNF$\alpha$; also lymphopenia (significantly reduced $\mathrm{CD} 4+$ and CD8+ T cells ) increased cytokine levels (e.g. IL-6, IL10 , and $\mathrm{TNF} \alpha$ ) associate with severity of COVID-19. In COVID-19 patients showed an increase in plasma levels of IL-1, IL-7, IL-8, IL-10, IL-17, IFN- $\gamma$,MCP-1, MIP-1A, MIP-1B, G-CSF, TNF- $\alpha$, inducible interferon protein (IP)-10 (Huang et al., 2020a; Qin et al., 2020). Cytokine storm can enhance viral sepsis and lung inflammation lead to pneumonitis; respiratory failure, organ failure, and possibly death. COVID-19 mortality is caused by multi-organ failure particularly in old age patients and individuals have chronic diseases such as cardiovascular disease, hypertension, and diabetes Prompetchara et al. (2020). The dysregulated immune response in COVID-19 patients can lead to a cytokine storm, causing increased respiratory disorder (Channappanavar and Perlman, 2017; Diao et al., 2020; Zhou et al., 2020) reported that 99 COVID-19 cases in Wuhan were investigated, increased total neutrophils (38\%), reduced total lymphocytes (35\%), increased serum IL-6 (52\%) and increased c-reactive protein (CRP) (84\%) were detected. The effective innate immune response against viral infection relies heavily on the interferon (IFN) type I responses and its downstream cascade that culminates in controlling 
viral replication and induction of effective adaptive immune response Shin et al. (2019). Lymphopenia appears in COVID -19 patients in severe cases this indicating immune system dysfunction Qin et al. (2020). From clinical studies during the COVID -19 crisis, patients with COVID-19 have a low level of helper T cells, cytotoxic T cells, and regulatory T cells; while $\mathrm{Th}$ cells and $\mathrm{T}$ reg. Cells are significantly decreased in severe patients Zhou et al. (2020). T regulatory (Treg.) cells are the responsible for conservation of the immune homeostasis with regulating the activation and proliferation of most lymphocytes such as T cytotoxic, Natural Killer (NK) cells, and B-lymphocytes Sakaguchi et al. (2010). In addition to the reduction of NK cells and Blymphocytes are reported in COVID-19 patients. From the above, these outcomes demonstrate that COVID-19 is responsible for an immune dysfunction with the induction of abnormal response of cytokine and chemokine, change in the level of lymphocytes subsets all of which might result in cytokine storm and tissue injury Wang et al. (2020). Cytokine storms can cause severe complications and worsens in COVID-19.

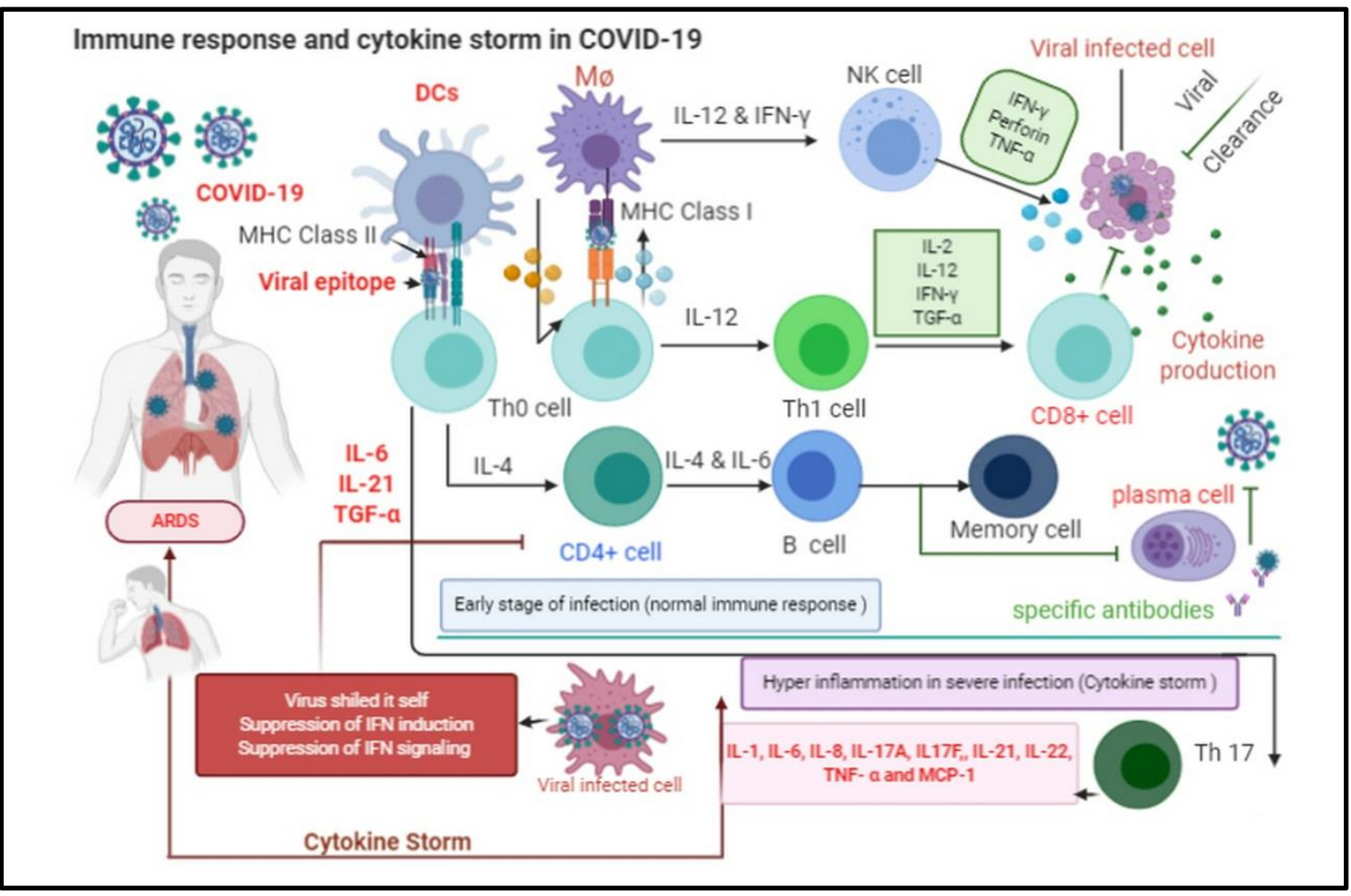

Fig.3: COVID-19 infected Dendritic cells (DCs); Macrophages (MФ), Epithelial cells are represent SARS CoV-2 epitope by MHC I to recruit T cytotoxic (CD8+T cell). Macrophage and dendritic cell (DC) are infected by SARS CoV-2 and represent SARS CoV-2 epitope by MHC II to recruit Thelper cells (CD4+T cell). Humoral immune response enhances the productionspecific antibody by plasma cell to SARS-CoV-2 epitope and viral neutralized. NK cells and CD8+T cells are secrete perforin and granzyme to kill virally infected cells (viral clearance). The antiviral activity of IFNs is vital in reducing the spreading of the virus. Some cytokines released by infected cells modulate the adaptive immune response by recruiting and activating immune cells such as B cells to regulate the removal of the virus. Further cytokine storm starts in severe cases of SARSCoV-2. Th17 secrets IL-1, IL-22, TNF- $\alpha$. Excessive inflammatory response means dysregulated immune response can lead to hyper inflammation causing severe worsen severe respiratory failure like acute respiratory distress syndrome (ARDS) and death in severe cases. 


\section{Conclusion}

In conclusion, the occurrence and development of SARS-CoV-2 depend on the interaction between the virus and the individual's immune system. Many factors correlated together, such as viral factors include virus type, viral load, mutation, and virus virulence. Also, the host immune system factors include human leukocyte antigen (HLA) genes or (MHC gene), gender, age, nutrition, immune regulation, and physical status. These elements all contribute to whether an individual is infected with the virus, the severity and duration of the illness, and the reinfection. Enormous inflammatory response with cytokine storm cause severe immunopathology and bad the prognosis in COVID-19. Definitely, drugs that directly target SARS-CoV-2 would be the most effective treatments for COVID-19. There are many protocols ongoing to find effective treatments for COVID-19 both targeting virus and consequent hyperinflammation. Treatment with antiviral agents alone may not be appropriate to stop the upsetting cytokine storm and pulmonary destruction in these patients. In early diagnosis stage of infection helps control the prevalence of the viral infection and treatment Patient follow up by successive analysis of acute COVID-19 samples to analyze innate and adaptive immunity would be useful to develop treatment strategies and prophylactic SARS-Cov-2 vaccines.

\section{References}

Addi, A.B., Lefort, A., Hua, X., Libert, F., Communi, D., Ledent, C., Macours, P., Tilley, S.L., Boeynaems, J.M., Robaye, B.J.E.j.o.i., 2008. Modulation of murine dendritic cell function by adenine nucleotides and adenosine: involvement of the $\mathrm{A} 2 \mathrm{~B}$ receptor. 38,1610 1620.
Al-Qahtani, A.A., Lyroni, K., Aznaourova, M., Tseliou, M., Al-Anazi, M.R., Al-Ahdal, M.N., Alkahtani, S., Sourvinos, G., Tsatsanis, C.J.O., 2017. Middle east respiratory syndrome corona virus spike glycoprotein suppresses macrophage responses via DPP4-mediated induction of IRAK-M and PPARY. 8, 9053.

Astuti, I.J.D., Research, M.S.C., Reviews, 2020. Severe Acute Respiratory Syndrome Coronavirus 2 (SARS-CoV-2): An overview of viral structure and host response.

Balachandran, S., Roberts, P.C., Brown, L.E., Truong, H., Pattnaik, A.K., Archer, D.R., Barber, G.N.J.I., 2000. Essential role for the dsRNA-dependent protein kinase PKR in innate immunity to viral infection. 13, 129-141.

Canton, J., Fehr, A.R., Fernandez-Delgado, R., Gutierrez-Alvarez, F.J., Sanchez-Aparicio, M.T., García-Sastre, A., Perlman, S., Enjuanes, L., Sola, I.J.P.p., 2018. MERS-CoV 4b protein interferes with the NF-KB-dependent innate immune response during infection. 14, e1006838.

Channappanavar, R., Perlman, S., 2017. Pathogenic human coronavirus infections: causes and consequences of cytokine storm and immunopathology. In: Seminars in immunopathology, pp. 529-539.

Cheng, V.C., Lau, S.K., Woo, P.C., Yuen, K.Y.J.C.m.r., 2007. Severe acute respiratory syndrome coronavirus as an agent of emerging and reemerging infection. 20,660-694.

Corman, V.M., Albarrak, A.M., Omrani, A.S., Albarrak, M.M., Farah, M.E., Almasri, M., Muth, D., Sieberg, A., Meyer, B., Assiri, A.M.J.C.I.D., 
2016. Viral shedding and antibody response in 37 patients with Middle East respiratory syndrome coronavirus infection. 62, 477-483.

Crespo, H.J., Lau, J.T., Videira, P.A.J.F.i.i., 2013. Dendritic cells: a spot on sialic acid. 4, 491.

Diao, B., Wang, C., Tan, Y., Chen, X., Liu, Y., Ning, L., Chen, L., Li, M., Liu, Y., Wang, G.J.F.i.I., 2020. Reduction and functional exhaustion of T cells in patients with coronavirus disease 2019 (COVID-19). 11, 827.

Hart, B.J., Dyall, J., Postnikova, E., Zhou, H., Kindrachuk, J., Johnson, R.F., Olinger Jr, G.G., Frieman, M.B., Holbrook, M.R., Jahrling, P.B.J.T.J.o.g.v., 2014. Interferon- $\beta$ and mycophenolic acid are potent inhibitors of Middle East respiratory syndrome coronavirus in cell-based assays. 95, 571.

He, Z., Zhao, C., Dong, Q., Zhuang, H., Song, S., Peng, G., Dwyer, D.E.J.I.j.o.i.d., 2005. Effects of severe acute respiratory syndrome (SARS) coronavirus infection on peripheral blood lymphocytes and their subsets. 9, 323-330.

Huang, C., Wang, Y., Li, X., Ren, L., Zhao, J., Hu, Y., Zhang, L., Fan, G., Xu, J., Gu, X., Cheng, Z., Yu, T., Xia, J., Wei, Y., Wu, W., Xie, X., Yin, W., Li, H., Liu, M., Xiao, Y., Gao, H., Guo, L., Xie, J., Wang, G., Jiang, R., Gao, Z., Jin, Q., Wang, J., Cao, B., 2020a. Clinical features of patients infected with 2019 novel coronavirus in Wuhan, China. Lancet 395, 497-506.

Huang, C., Wang, Y., Li, X., Ren, L., Zhao, J., Hu, Y., Zhang, L., Fan, G., Xu, J., Gu, X.J.T.I., 2020b. Clinical features of patients infected with 2019 novel coronavirus in Wuhan, China. 395, 497506.
Kato, H., Takahasi, K., Fujita, T.J.I.r., 2011. RIG-I-like receptors: cytoplasmic sensors for non-self RNA. $243,91-98$.

Killerby, M.E., Biggs, H.M., Haynes, A., Dahl, R.M., Mustaquim, D., Gerber, S.I., Watson, J.T.J.J.o.C.V., 2018. Human coronavirus circulation in the United States 2014-2017. 101, 52-56.

Lam, C.W., Chan, M.H., Wong, C.K.J.T.C.B.R., 2004. Severe acute respiratory syndrome: clinical and laboratory manifestations. 25, 121.

Li, C.K.-f., Wu, H., Yan, H., Ma, S., Wang, L., Zhang, M., Tang, X., Temperton, N.J., Weiss, R.A., Brenchley, J.M.J.T.J.o.I., 2008. T cell responses to whole SARS coronavirus in humans. 181, 5490-5500.

Li, C.K.-f., Xu, X., 2010. Host Immune Responses to SARS Coronavirus in Humans, In: Molecular biology of the SARS-coronavirus. Springer, pp. 259-278.

Li, G., Fan, Y., Lai, Y., Han, T., Li, Z., Zhou, P., Pan, P., Wang, W., Hu, D., Liu, X.J.J.o.m.v., 2020. Coronavirus infections and immune responses. 92, 424-432.

Maclachlan, N.J., Dubovi, E.J., 2010. Fenner's veterinary virology. Academic press.

Mahallawi, W.H., Khabour, O.F., Zhang, Q., Makhdoum, H.M., Suliman, B.A.J.C., 2018. MERS-CoV infection in humans is associated with a pro-inflammatory Th1 and Th17 cytokine profile. 104, 8-13.

Manni, M.L., Robinson, K.M., Alcorn, J.F.J.E.r.o.r.m., 2014. A tale of two cytokines: IL-17 and IL-22 in asthma and infection. 8, 25-42. 
Mathern, D.R., Heeger, P.S.J.C.J.o.t.A.S.o.N., 2015. Molecules great and small: the complement system. 10, 1636-1650.

Mubarak, A., Alturaiki, W., Hemida, M.G.J.J.o.i.r., 2019. Middle east respiratory syndrome coronavirus (MERS-CoV): infection, immunological response, and vaccine development. 2019.

Nicholls, J.M., Poon, L.L., Lee, K.C., Ng, W.F., Lai, S.T., Leung, C.Y., Chu, C.M., Hui, P.K., Mak, K.L., Lim, W.J.T.L., 2003. Lung pathology of fatal severe acute respiratory syndrome. 361, 1773-1778.

Prompetchara, E., Ketloy, C., Palaga, T.J.A.P.J.A.I., 2020. Immune responses in COVID-19 and potential vaccines: Lessons learned from SARS and MERS epidemic. 38, 1-9.

Qin, C., Zhou, L., Hu, Z., Zhang, S., Yang, S., Tao, Y., Xie, C., Ma, K., Shang, K., Wang, W., Tian, D.-S., 2020. Dysregulation of Immune Response in Patients With Coronavirus 2019 (COVID-19) in Wuhan, China. Clinical Infectious Diseases.

Risitano, A.M., Mastellos, D.C., Huber-Lang, M., Yancopoulou, D., Garlanda, C., Ciceri, F., Lambris, J.D.J.N.R.I., 2020. Complement as a target in COVID-19? 20, 343-344.

Sakaguchi, S., Miyara, M., Costantino, C.M., Hafler, D.A.J.N.R.I., 2010. FOXP3+ regulatory T cells in the human immune system. 10, 490-500.

Schulze, K., Staib, C., Schätzl, H.M., Ebensen, T., Erfle, V., Guzman, C.A.J.V., 2008. A prime-boost vaccination protocol optimizes immune responses against the nucleocapsid protein of the SARS coronavirus. 26, 6678-6684.

Sevajol, M., Subissi, L., Decroly, E., Canard, B., Imbert, I.J.V.r., 2014. Insights into RNA synthesis, capping, and proofreading mechanisms of SARS-coronavirus. 194, 90-99.

Shin, H.-S., Kim, Y., Kim, G., Lee, J.Y., Jeong, I., Joh, J.S., Kim, H., Chang, E., Sim, S.Y., Park, J.S.J.C.I.D., 2019. Immune responses to Middle East respiratory syndrome coronavirus during the acute and convalescent phases of human infection. 68, 984-992.

Trapani, J.A.J.G.b., 2001. Granzymes: a family of lymphocyte granule serine proteases. 2, reviews3014. 3011.

Tufan, A., GÜLER, A.A., Matucci-Cerinic, M.J.T.J.o.M.S., 2020. COVID-19, immune system response, hyperinflammation and repurposing antirheumatic drugs. 50, 620-632.

Wang, F., Nie, J., Wang, H., Zhao, Q., Xiong, Y., Deng, L., Song, S., Ma, Z., Mo, P., Zhang, Y., 2020. Characteristics of Peripheral Lymphocyte Subset Alteration in COVID-19 Pneumonia. The Journal of Infectious Diseases 221, 17621769.

Welsh, R.M., Waggoner, S.N.J.V., 2013. NK cells controlling virus-specific T cells: Rheostats for acute vs. persistent infections. 435, 37-45.

Wong, C., Lam, C., Wu, A., Ip, W., Lee, N., Chan, I., Lit, L., Hui, D., Chan, M., Chung, S.J.C., Immunology, E., 2004. Plasma inflammatory cytokines and chemokines in severe acute respiratory syndrome. 136, 95-103.

Xiao, K., Zhai, J., Feng, Y., Zhou, N., Zhang, X., Zou, J.-J., Li, N., Guo, Y., Li, X., Shen, X.J.B., 2020. Isolation and characterization of 2019-nCoVlike coronavirus from Malayan pangolins.

Xie, J., Han, Y., Li, T., Qiu, Z., Ma, X., Fan, H., Lü, W., Liu, Z., Wang, Z., Wang, H.J.Z.n.k.z.z., 2003. 
Dynamic changes of plasma cytokine levels in patients with severe acute respiratory syndrome. 42, 643-645.

Xu, X., Gao, X.-M.J.C.M.I., 2004. Immunological responses against SARS-coronavirus infection in humans. 1, 119-122.

Yang, Y., Lu, Q., Liu, M., Wang, Y., Zhang, A., Jalali, N., Dean, N., Longini, I., Halloran, M.E., Xu, B.J.M., 2020. Epidemiological and clinical features of the 2019 novel coronavirus outbreak in China.

Yi, Y., Lagniton, P.N., Ye, S., Li, E., Xu, R.-H.J.I.j.o.b.s., 2020. COVID-19: what has been learned and to be learned about the novel coronavirus disease. 16, 1753.

Ying, T., Li, W., Dimitrov, D.S. 2016. Discovery of T-cell infection and apoptosis by Middle East respiratory syndrome coronavirus (Oxford University Press).

Zhao, J., Li, K., Wohlford-Lenane, C., Agnihothram, S.S., Fett, C., Zhao, J., Gale, M.J., Baric, R.S., Enjuanes, L., Gallagher, T.J.P.o.t.N.A.o.S.,
2014. Rapid generation of a mouse model for Middle East respiratory syndrome. 111, 49704975.

Zhou, J., Chu, H., Chan, J.F.-W., Yuen, K.-Y.J.V.j., 2015. Middle East respiratory syndrome coronavirus infection: virus-host cell interactions and implications on pathogenesis. 12, 218.

Zhou, J., Chu, H., Li, C., Wong, B.H.-Y., Cheng, Z.-S., Poon, V.K.-M., Sun, T., Lau, C.C.-Y., Wong, K.K.Y., Chan, J.Y.-W.J.T.J.o.i.d., 2014. Active replication of Middle East respiratory syndrome coronavirus and aberrant induction of inflammatory cytokines and chemokines in human macrophages: implications for pathogenesis. 209, 1331-1342.

Zhou, Y., Fu, B., Zheng, X., Wang, D., Zhao, C., Qi, Y., Sun, R., Tian, Z., Xu, X., Wei, H.J.B., 2020. Aberrant pathogenic GM-CSF+ T cells and inflammatory CD14+ CD16+ monocytes in severe pulmonary syndrome patients of a new coronavirus. 